Annuaire suisse de politique de développement

$17 \mid 1998$

Propriété intellectuelle : quels enjeux pour les pays en développement ?

\title{
3. Le commerce mondial
}

\section{CpenEdition}

\section{Journals}

Édition électronique

URL : http://journals.openedition.org/aspd/770

DOI : $10.4000 /$ aspd. 770

ISSN : 1663-9669

Éditeur

Institut de hautes études internationales et du développement

Édition imprimée

Date de publication : 1 avril 1998

Pagination : 171-189

ISSN : 1660-5934

Référence électronique

《3. Le commerce mondial », Annuaire suisse de politique de développement [En ligne], 17| 1998, mis en ligne le 01 avril 1998, consulté le 07 septembre 2020. URL : http://journals.openedition.org/aspd/770 DOI : https://doi.org/10.4000/aspd.770 


\section{LE COMMERCE MONDIAL}

\subsection{LE COMMERCE MONDIAL EN 1996'}

\section{Croissance en volume de la production}

et des exportations mondiales de marchandises

En 1996, le commerce mondial des biens a augmenté de 4\% en volume, soit une progression analogue à celle observée pendant la période 1990-1993. Ce ralentissement fait suite, comme le montre le graphique ci-dessous, à deux années de croissance exceptionnelle du volume des échanges mondiaux (1994 et 1995). Le taux de croissance de la production mondiale des biens a un peu baissé pour la deuxième année consécutive, mais est resté très supérieur aux taux enregistrés pendant les quatre premières années de la décennie (graphique $\mathrm{n}^{\circ} 1$ ).

\section{Graphique n 1}

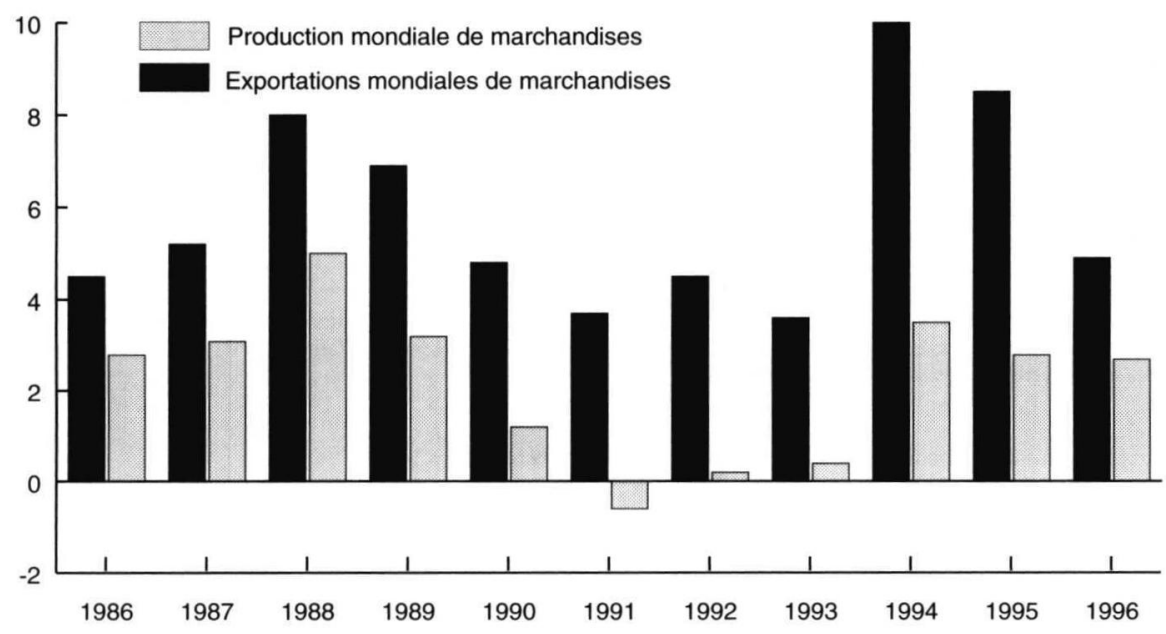

Source: Organisation mondiale du commerce, Communiqué de presse, Press/71, avril 1997, «Après deux années exceptionnelles, la croissance du commerce mondial revient en 1996 à son rythme antérieur», p. 6.

1. Cette introduction met en lumière les tendances et l'évolution du commerce international en 1996. Ces informations proviennent du rapport annuel de l'Organisation mondiale du commerce 1996 et d'une actualisation des données publiées en avril 1997. 
Les exportations de biens ont pour la première fois passé la barre des 5000 milliards de dollars. Elles se sont chiffrées à 5100 milliards de dollars exactement, en progression de $4 \%$. Certes, le ralentissement de la croissance en volume a contribué à la forte décélération en valeur - de près de $20 \%$ en 1995 à $4 \%$ en 1996 -, mais celle-ci s'explique principalement par l'effet de l'appréciation du dollar l'année dernière à l'égard des monnaies des autres grandes nations commerçantes.

L'OMC estime que la valeur des exportations mondiales de services commerciaux a augmenté de $5 \%$ en 1996, contre $14 \%$ en 1995 . Le ralentissement était particulièrement prononcé en Europe occidentale et en Asie. En partie à cause des effets de change, le taux de croissance du commerce deservices commerciaux de ces deux régions a diminué de deux tiers en 1996. Malgré ce fléchissement, les exportations de services atteignent elles aussi un nouveau sommet, puisqu'elles sont évaluées à 1200 milliards de dollars au total.

Tableau $\mathbf{n}^{\circ} 12$

Croissance en volume des exportations mondiales de marchandise et de services commerciaux

(en milliards de dollars et en pourcentage)

\begin{tabular}{lcccr}
\hline & \multicolumn{2}{c}{$\begin{array}{c}\text { Valeur } \\
\text { (milliards de dollars) }\end{array}$} & \multicolumn{2}{c}{$\begin{array}{c}\text { Variation annuelle } \\
\text { (pourcentage) }\end{array}$} \\
\hline Marchandises & $\mathbf{1 9 9 5}$ & $\mathbf{1 9 9 6}$ & $\mathbf{1 9 9 5}$ & $\mathbf{1 9 9 6}$ \\
\hline Services commerciaux & 4920 & 5115 & 19.5 & 4.0 \\
\hline
\end{tabular}

Note: les données relatives aux exportations de services commerciaux et aux exportations de marchandises ne sont pas directement comparables, essentiellement parce que les premières proviennent des statistiques de la balance des paiements et les secondes des statistiques douanières.

Source: Organisation mondiale du commerce, Rapport annuel 1997, vol. I, p.14.

\section{$\square$ Commerce mondial des marchandises par régions}

Dans chacune des sept régions géographiques employées dans les statistiques de l'OMC, la croissance du volume des exportations de marchandises a été moins prononcée en 1996 qu'en 1995. Le taux de croissance des exportations de l'Europe occidentale, de l'Asie et des pays en transition a été compris entre le quart et la moitié des taux enregistrés en 1995, mais la décélération a été moins marquée en Amérique du Nord et minime en Amérique latine. 
Tableau n ${ }^{\circ} 13$

Croissance en volume du commerce mondial des marchandises dans certaines régions, 1990-1996

(variation en pourcentage)

\begin{tabular}{|c|c|c|c|c|c|c|}
\hline \multicolumn{3}{|c|}{ Exportations } & & \multicolumn{3}{|c|}{ Importations } \\
\hline 1990-96 & 1995 & 1996 & & $0-96$ & 1995 & 1996 \\
\hline 6.0 & 8.5 & 5.0 & Monde entier & 6.0 & 9.0 & 5.0 \\
\hline 7.0 & 9.5 & 6.0 & Amérique du Nord' & 7.0 & 8.0 & 5.5 \\
\hline 8.5 & 12.0 & 10.5 & Amérique latine & 11.5 & 3.0 & 10.5 \\
\hline 5.0 & 7.5 & 4.5 & Europe occidentale & 4.0 & 7.0 & 3.5 \\
\hline 5.5 & 7.5 & 4.0 & Union européenne (15) & 4.0 & 6.5 & 3.5 \\
\hline 7.0 & 9.5 & 4.0 & Asie & 9.5 & 14.0 & 5.0 \\
\hline 1.0 & 3.5 & -0.5 & Japon & 5.5 & 12.5 & 2.5 \\
\hline 10.5 & 14.5 & 7.0 & $\begin{array}{l}\text { Six pays exportateurs } \\
\text { d'Asie de l'Est }^{2}\end{array}$ & 11.0 & 15.0 & 5.0 \\
\hline 1.5 & 2.5 & 8 & Afrique* & 3.5 & 11.5 & 6.0 \\
\hline 4.5 & 2.5 & 2.5 & Moyen-Orient* & 5.1 & 2.0 & 9.5 \\
\hline
\end{tabular}

1. Canada et Etats-Unis.

2. Hongkong, Chine, Malaisie, République de Corée, Singapour, Taipei chinois et Thaïlande.

* Données non officielles.

Source des données officielles: Organisation mondiale du commerce, Rapport annuel de l'OMC 997, vol. I, p.19.

- La croissance du commerce des pays d'Asie se ralentit plus qu'ailleurs. L'écart s'est réduit en 1996, en grande partie par suite de l'évolution des flux commerciaux en Asie, où la croissance en volume des exportations et des importations a été inférieure à celle de la production. Le Japon est le pays dans lequel il y a eu la plus forte divergence entre la croissance des exportations et celle des importations durant cette période: en volume, ses importations ont progressé six fois plus que ses exportations.

๖ D'autres régions réalisent une meilleure performance au niveau commercial: en Amérique du Nord, en Amérique latine et en Europe occidentale, la croissance du volume des échanges est restée au moins deux fois supérieure à celle du PIB.

L'expansion exceptionnelle des exportations de l'Amérique latine peut être attribuée en grande partie au Mexique, dont les exportations en volume ont augmenté de plus de $20 \%$ pour la deuxième année consécutive.

๖ Bien que les économies en transition aient enregistré des résultats supérieurs à la moyenne au cours des deux dernières années, ces pays sont ceux dont les exportations et les importations ont augmenté le moins rapidement depuis 1990. De plus ces économies font face à une nouvelle contraction de la production. 
- Différences régionales: l'Amérique latine et le Moyen-Orient ${ }^{2}$ ont enregistré une croissance de $10 \%$ ou plus en valeur de leurs exportations et importations de biens. Sur les sept régions géographiques, ce sont les économies en transition qui ont connu la croissance la plus rapide de leurs importations.

- L'Afrique dépasse la moyenne mondiale: pour la première fois depuis 1990, les exportations et importations de l'Afrique ont progressé plus rapidement en valeur que le commerce mondial total, en grande partie du fait de l'augmentation des exportations de combustibles.

\section{$\square$ Commerce des pays les moins avancés}

Les statistiques commerciales communiquées par les PMA pour 1996 sont extrêmement lacunaires et celles de leur principale partenaire commerciale - l'Union européenne - sont encore incomplètes. Malgré l'évolution généralement défavorable des prix des produits primaires et la faiblesse des importations de l'Europe occidentale, les quelques pays d'Afrique dont on possède des données ont accru leurs exportations de $5 \%$ à $12 \%$ (en dollars) en 1996. Les deux seuls PMA exportateurs de pétrole (Angola et Yémen) ont bénéficié de la fermeté du prix du pétrole.

\section{$\square$ Commerce mondial des marchandises par produits}

En 1996, la croissance nominale des échanges a été inférieure à celle de 1995 pour tous les groupes de produits, à l'exception des combustibles. Le mouvement de décélération a été plus ou moins marqué selon les groupes. Les produits primaires, qui sont très sensibles aux fluctuations cycliques de la demande, ont enregistré les variations les plus importantes pour ce qui est des prix en dollars et de la valeur.

Comme le montre le graphique $\mathrm{n}^{\circ} 2$ ci-après, en 1996, les exportations mondiales de combustibles ont augmenté de 18,5\% tandis que les expéditions de matières premières agricoles et de métaux non ferreux ont diminué de plus de 5\%. Parmi les produits manufacturés, seuls le fer et l'acier ont vu la valeur de leurs exportations baisser en 1996; les taux de croissance des autres groupes de produits ont sensiblement diminué, mais sont restés positifs. La croissance des expéditions de matériels de bureau et de télécommunication, qui étaient de loin les plus dynamiques de toutes les exportations mondiales de marchandises pendant la dernière décennie, a accusé une forte baisse, revenant à 30\% en 1995 et à environ $4 \%$ en 1996. Les échanges de vêtements et d'autres biens de consommation ainsi que des produits de l'industrie automobile et d'autres machines ont progressé de $4 \%$. D'après les estimations les exportations mondiales de textile auraient stagné ${ }^{3}$.

ASTM 98, «Statistiques», chapitre sur le commerce, p. 349.

2. Sous l'effet de la hausse sensible des prix du pétrole, la valeur des exportations de combustibles a progressé de plus de $10 \%$ en 1996, ce qui est le plus gros gain enregistré depuis 1990 (29\%).

3. Organisation mondiale du commerce, Rapport annuel de l'OMC 1997, vol. I et II, Genève, OMC, décembre 1997, pp. 14 et 15. 


\section{Graphique $\mathbf{n}^{\circ} 2$ \\ Croissance en valeur du commerce mondial des marchandises, par groupe de produits, 1996 \\ (variation en pourcentage)}

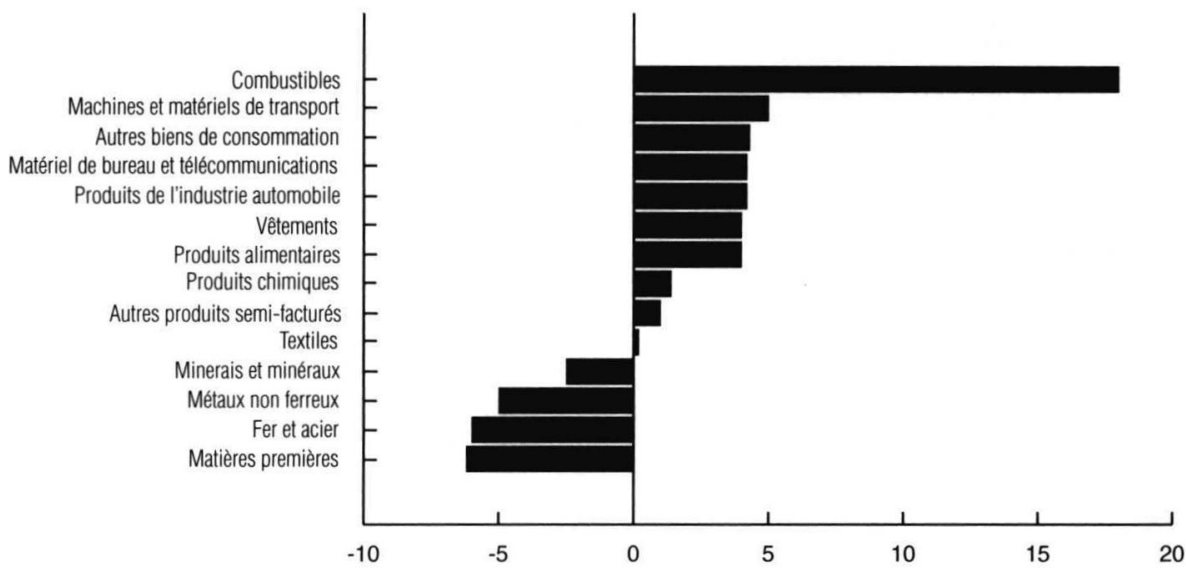

1. Non compris les produits de l'industrie automobile et les matériels de bureau et de télécommunications.

Source: Organisation mondiale du commerce, Rapport annuel de l'OMC 1997, vol. I, p.15.

\section{SOURCES}

Organisation mondiale du commerce, Rapport annuel de l'OMC 1996, vol. I et II, Genève, OMC, décembre 1996. Organisation mondiale du commerce, Rapport annuel de l'OMC 1997, vol. I et II, Genève, OMC, décembre 1997.

Organisation mondiale du commerce, communiqué de presse «Après deux années exceptionnelles, la croissance du commerce mondial revient en 1996 à son rythme antérieur», avril 1997 - Press/71.

Organisation mondiale du commerce, Communiqué de presse, Press/71, avril 1997, «Après deux années exceptionnelles, la croissance du commerce mondial revient en 1996 à son rythme antérieur».

\subsection{ORGANISATION MONDIALE DU COMMERCE}

En 1997 trois importants accords internationaux ont été conclus dans le cadre de l'Organisation mondiale du commerce (accords sur la libéralisation des télécommunications, sur les technologies de l'information et sur les services financiers). L'OMC a également poursuivi l'étude des demandes d'accession qui ont abouti à l'adhésion de quatre nouveaux membres. Afin de répondre aux demandes des ministres présents lors de la $1^{\text {re }}$ Conférence des Etats membres à Singapour en décembre 1996, l'OMC a organisé en octobre 1997 une importante réunion consacrée à la mise au point d'une stratégie pour une meilleure intégration des pays les moins avancés dans le commerce international. La Suisse, membre de l'OMC, a signé les trois accords et a pris les mesures législatives pour qu'ils soient compatibles avec le droit suisse. De plus elle s'est particulièrement engagée dans les travaux préparatoires et lors de la Conférence sur l'intégration des PMA au commerce international.

Instituée le $1^{\text {er }}$ janvier 1995 à la suite des négociations de l'Uruguay Round, l'OMC a son siège à Genève. Le secrétariat, dont le directeur général est Renato 
Ruggiero, occupe environ 500 personnes. Le budget annuel de l'organisation, qui s'est monté à environ 93 millions de dollars en 1996, est alimenté par les contributions de chaque membre au prorata de sa part dans le volume total des échanges. La contribution de la Suisse s'est montée à 2 millions de francs suisses en 1996.

L'OMC a six fonctions essentielles:

• gérer et surveiller les 28 accords multilatéraux et plurilatéraux qui constituent le traité de l'OMC;

- constituer un forum pour les négociations multilatérales internationales;

- fournir des mécanismes permettant de résoudre les conflits commerciaux de ses membres;

- examiner et évaluer les politiques commerciales de ses membres;

- coopérer avec d'autres organisations internationales importantes impliquées dans la gestion de l'économie globale;

- aider les économies en développement et en transition économique à mieux s'intégrer dans le système commercial multilatéral.

\section{$\square$ Pays membres et nouvelles accessions}

Parmi les activités de l'OMC figure l'accroissement du nombre de ses membres. Depuis l'établissement de l'Organisation, quatre pays ont achevé leur processus d'accession (Equateur, Bulgarie, Mongolie et Panama). Tous les pays de l'Amérique latine sont maintenant membres. En novembre 1997, le nombre des membres s'élevait à 132 pays. La plupart sont d'anciens membres du GATT qui ont signé l'Acte final de l'Uruguay Round et mené à bien les procédures de ratification. Afin d'évaluer les demandes d'adhésion, l'OMC a constitué 28 groupes de travail chargés de conduire les pourparlers avec les pays souhaitant y accéder. Une trentaine de pays ont fait parvenir une demande d'accession. Certains sont des acteurs jouant un rôle de premier plan sur la scène économique et commerciale ou figurent parmi les marchés émergents les plus importants. Il s'agit notamment de pays tels l'Arabie Saoudite, la République populaire de Chine, la Fédération de Russie, Taiwan. De nombreux pays en transition figurent également sur les rangs: Albanie, Arménie, Azerbaïdjan, Biélorussie, Croatie, Estonie, Géorgie, Kazakhstan, Lettonie, Lituanie, ex-République yougoslave de Macédoine, Moldavie, Ouzbékistan, République Kirghize, Ukraine, Vietnam.

De l'avis même de l'OMC ${ }^{4}$, «la forte volonté de ces pays de se joindre à l'OMC montre l'attrait croissant que suscite l'idée d'un système commercial vraiment universel basé sur des règles convenues au niveau international avec les moyens voulus pour les faire respecter».

A noter la situation particulière de la Chine et de Hongkong. Depuis le $1^{\text {er }}$ juillet 1997, la Chine exerce sa pleine souveraineté sur Hongkong. Cependant, suivant le principe «un pays, deux systèmes», les règles de fonctionnement spécifiques de l'économie de marché de Hongkong semblent préservées, ainsi que son statut de membre à part entière au sein de l'OMC en tant que «région administrative spéciale». Hongkong avait reçu le statut de membre du GATT à titre de «territoire douanier séparé de la Grande-Bretagne» en 1986. Un an plus tard la Chine,

4. Organisation mondiale du commerce, Rapport annuel 1996, vol. I, Genève, OMC, 1996, p. 7. 
qui a un statut d'observateur, déposait sa candidature, enjeu de difficiles négociations sur les conditions de son admission. Les pays occidentaux lui reprochent des tarifs douaniers élevés et un marché protégé, et l'attribution de subventions pour certaines industries. La Communauté européenne et les Etats-Unis refusent d'accorder le statut de pays en développement à la Chine, ce qui lui laisserait un délai plus long pour se conformer aux critères d'adhésion (allégement de tarifs douaniers et ouverture du marché).

\section{Accords commerciaux conclus en 1997}

\section{Accord sur la libéralisation des télécommunications}

Les négociations de l'OMC sur les télécommunications se sont conclues le 15 février 1997. Soixante-neuf gouvernements ont pris des engagements multilatéraux en vue de libéraliser les échanges dans ce secteur concernant un marché dont la valeur dépasse largement 550 milliards de dollars par an. Ces pays représentent plus de $90 \%$ des recettes tirées des télécommunications au niveau mondial. Les négociations ont commencé en mars 1994 et devaient se terminer au plus tard en avril 1996. Aucun accord n'ayant été conclu à cette date, les pourparlers ont été prolongés et c'est grâce à l'impulsion des ministres réunis à Singapour que les négociations ont pu s'achever en février 1997. Pendant cette période de nombreux pays ont révisé leur offre. La Suisse, par exemple, a dû s'engager à la pleine ouverture de son marché au $1^{\text {er }}$ janvier $1998^{5}$. L'accord couvre l'ensemble des services de télécoms ainsi que les techniques de transmission et s'appuie principalement sur la suppression des monopoles d'Etat. Cette situation nouvelle va intensifier la concurrence dans les Etats signataires et unifier les règles du jeu pour les fournisseurs qui investissent hors de leurs frontières.

Selon le directeur général de l'OMC $\mathrm{OM}^{6}$, Renato Ruggiero, "l'accord sur les télécommunications contribuera à abaisser le coût pour le consommateur et les réductions de prix seront très importantes. Les entreprises dépensent globalement plus pour les services des télécommunications que pour le pétrole». Il a ajouté que «la libéralisation des télécommunications pourrait représenter un gain de revenu global d'environ 1000 milliards de dollars au cours des dix prochaines années, ce qui équivaut à environ $4 \%$ du PIB mondial aux prix actuel».

Les négociations de libéralisation au niveau mondial signifient une ouverture à la concurrence internationale d'un large éventail de services de télécommunication. Bien que la plupart des pays en développement participant aux négociations aient, individuellement, contribué pour une faible part aux télécommunications mondiales, ils connaissent une croissance beaucoup plus rapide dans ce secteur que les pays développés:

- pour les lignes téléphoniques principales le taux de croissance annuel moyen dans les pays développés n'a été que de 3,5\% pendant la période de 1990 à 1995, alors qu'il a dépassé $13,8 \%$ en moyenne dans les pays en développement;

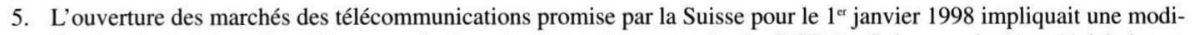
fication de la loi sur les télécommunications basée sur le monopole des PTT. La Suisse a adapté sa législation et adopté une nouvelle loi sur les télécommunications (LTC). La nouvelle loi est entrée en vigueur le 1" janvier 1998, ce qui permet à la Suisse de respecter ses engagements internationaux.

6. Organisation mondiale du commerce, Focus, n 16, février 1997, p. 1. 
- pour les recettes tirées des télécommunications, la croissance annuelle moyenne des pays développés a été de 4,2\% (période 1990-1995). Pendant la même période la croissance dans les pays en développement a été plus de deux fois supérieure, se situant à $9,7 \%$.

Toutefois, pour certains observateurs asiatiques ${ }^{7} l^{\prime}$ accord de l'OMC va surtout profiter aux entreprises américaines qui sont bien placées pour dominer le marché.

La Société pour le développement de l'économie suisse ${ }^{8}$ s'est prononcée en faveur d'une libéralisation rapide des télécommunications, tout en soulignant son importance décisive pour l'attractivité et la compétitivité de l'économie suisse.

En Suisse, la libéralisation du marché des télécommunications a amené la nouvelle société Swisscom à une restructuration dont le coût se traduit notamment par une réduction d'effectif de $21^{\prime} 500$ à $17^{\prime} 000$ postes de travail d'ici à $2001^{\prime}$.

\section{Accord sur les technologies de l'information}

Une Déclaration ministérielle sur le commerce des produits des technologies de l'information (ATI) avait été adoptée à l'issue de la première conférence ministérielle de l'OMC en décembre 1996 à Singapour. Le 26 mars 1997, 40 gouvernements sont convenus de mettre en œuvre cette Déclaration en vertu de laquelle les droits de douane et autres impositions frappant les produits des technologies de l'information (ordinateurs, matériels de télécommunication, semiconducteurs, logiciels) seront progressivement réduits pour être complètement éliminés en l'an 2000. Les pays industrialisés, sans exception, se sont engagés à ramener leurs droits de douane à zéro d'ici à l'an 2000. Plusieurs pays en développement, parmi lesquels l'Inde, Taiwan, la Malaisie, l'Indonésie, la Thaïlande et la Corée du Sud (qui figurent parmi les importants exportateurs des produits des technologies de l'information), ont toutefois jusqu'en 2005 pour supprimer leurs droits de douane sur certaines marchandises spécifiques. Selon Renato Ruggiero, «cet accord, qui couvre des échanges mondiaux d'une valeur proche de 600 milliards de dollars, implique un abaissement des prix pour les consommateurs et une réduction des obstacles entravant la diffusion de la technologie (...)».

La Suisse, signataire de l'accord, a mis en œuvre les réductions tarifaires dès le 31 décembre 1997. Elle ramènera de manière graduelle ses droits de douane à

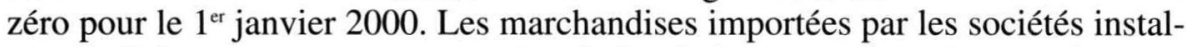
lées en Suisse ne seront plus soumises à des droits de douane, alors que les produits des entreprises helvétiques deviendront moins chers à l'étranger. En 1996, le montant des droits de douane prélevés par la Confédération sur les produits couverts par l'accord s'est élevé à 24 millions de francs.

Les deux accords (sur la libéralisation des télécommunications et sur les technologies de l'information) portent sur des transactions internationales d'une valeur supérieure à 1000 milliards de dollars (évaluation de l'OMC), ce qui correspond à peu près au commerce mondial des produits agricoles (444 milliards de dollars), des automobiles (456 milliards de dollars) et des textiles (153 milliards de dollars) considérés ensemble. En novembre 1997, le Conseil fédéral a annoncé la ratification par la Suisse de l'Accord sur la libéralisation des télécommunica-

7. Far Eastern Economic Review, 27 February 1997 «Fax Americana».

8. Société pour le développement de l'économie suisse, Opinion, «Pour une libéralisation des télécommunications", $\mathrm{n}^{\circ} 9 \mathrm{a}, 25$ février 1997.

9. Journal de Genève, 24 octobre 1997 «Swisscom se prépare à la libéralisation et supprimera 1100 emplois en 1998». 
tions et de l'Accord sur les technologies de l'information. L'entrée en vigueur de la réglementation du secteur des télécommunications coïncide avec la libéralisation du marché suisse.

\section{Accord sur la libéralisation des services financiers}

Le 12 décembre 1997 un accord libéralisant les activités bancaires et les assurances dans le monde a été conclu sous les auspices de l'OMC. Après une difficile année de négociations, 70 Etats membres de l'OMC, dont la Suisse, se sont engagés à améliorer et à garantir l'accès de leur marché des banques et des assurances à la concurrence étrangère. L'accord sur les services financiers couvre plus de $95 \%$ du marché des banques, des assurances, des titres et de l'information financière. Selon l'OMC ce secteur représente des milliers de milliards de dollars ${ }^{10}$. Les activités dans les services financiers ont explosé ces dix dernières années, pour représenter aujourd'hui un volume d'affaires de 140'000 millions de dollars, soit 20 fois plus que le PIB américain ${ }^{11}$. L'accord rentrera en vigueur le $1^{\text {er }}$ mars 1999 au plus tard.

La conclusion de cet accord était fortement désirée par la Suisse qui détient en la matière un très fort potentiel. L'Association suisse des banquiers (ASB) a réagi en accueillant favorablement la signature du traité. Selon elle, les exportateurs et sociétés de services indigènes pourront encore davantage être aidés par les groupes financiers suisses qui sont en place dans les pays signataires de l'accord ${ }^{12}$.

\section{$\square$ Réunion de haut niveau pour favoriser le commerce avec les PMA}

A la demande des ministres présents lors de la Conférence ministérielle à Singapour, l'OMC a organisé, en partenariat avec d'autres institutions internationales ${ }^{13}$, une «réunion de haut niveau sur la mise en place de mesures intégrées en faveur du développement du commerce pour les pays les moins avancés». La conférence s'est tenue les 27 et 28 octobre 1997 à Genève. Lors du discours inaugural, Renato Ruggiero a rappelé que les PMA représentent $10 \%$ de la population mondiale mais ne participent qu'à 0,5 à $1 \%$ du commerce international. Les investissements étrangers directs y sont insignifiants.

La Suisse, dont l'un des objectifs déclarés de sa politique au développement est l'intégration des PMA au commerce international, s'est beaucoup engagée dans l'organisation de l'événement (elle a notamment participé à son financement pour un montant de 250'000 francs). Elle a également activement collaboré aux travaux préparatoires. La délégation suisse, conduite par le secrétaire d'Etat Franz Blankart, était composée de représentants de l'Office fédéral des affaires économiques extérieures, de la Mission suisse près de l'OMC et la CNUCED, de la DDC, ainsi que d'un représentant de la Communauté de travail des œuvres d'entraide.

La mise au point d'une stratégie pour une meilleure intégration des pays les moins avancés dans le commerce international était l'objectif central de la conférence. Deux thèmes principaux ont été abordés: l'accès au marché et la défini-

10. Organisation mondiale du commerce, Communiqué de presse, Press/86, 15 décembre 1997 «Succès des négociations de l'OMC sur les services financiers".

11. Journal de Genève, 15 décembre 1997 «Un accord a été signé sur la libéralisation des services financiers».

12. L'Agefi, 12 décembre 1997 «L'OMC a réussi la libéralisation des services financiers».

13. Cette réunion a été organisée par l'OMC, en collaboration avec la CNUCED, le Centre de commerce international (CCI), la Banque mondiale, le Fonds monétaire international et le PNUD. 
tion d'une approche intégrée de coopération technique dans le domaine du commerce. Parallèlement une douzaine de tables rondes ont été organisées pendant lesquelles les PMA choisis ont pu exprimer leurs besoins en matière de coopération technique. Enfin deux tables rondes thématiques, la première sur la capacité d'échanges des PMA, la seconde consacrée à l'encouragement des investissements dans les PMA, ont abouti à une série de recommandations ${ }^{14}$.

\section{Amélioration de l'accès aux marchés pour les PMA}

Les pays industrialisés et quelques pays en développement présents lors de la conférence ont annoncé des mesures nouvelles ou additionnelles concernant l'accès préférentiel aux marchés pour les PMA. Concernant ce point, le secrétaire d'Etat Franz Blankart a rappelé la révision du schéma de préférences douanières suisses, qui est entré en vigueur en mars 1997 et permet notamment un libre accès au marché helvétique pour les $98 \%$ des exportations des PMA ${ }^{15}$.

\section{Adoption d'une approche intégrée de coopération technique}

Le cadre intégré ${ }^{16}$ vise à accroître la cohérence et l'efficacité dans la définition et la mise en œuvre des programmes nationaux d'assistance technique et financière destinés à promouvoir l'intégration des PMA dans le commerce international. Les six organisations internationales concernées sont l'OMC, la CNUCED, le Centre de commerce international (CCI), la Banque mondiale, le Fonds monétaire international et le PNUD.

Les activités d'assistance technique liées au commerce fournies par les organisations impliquées comprennent notamment:

- le renforcement des capacités institutionnelles en vue de traiter les questions de politique commerciale (aider les PMA à définir une politique commerciale, les assister dans leur demande d'accession à l'OMC, favoriser l'accès à l'information pour négocier sur des questions commerciales);

- l'accroissement des capacités d'exportation (faciliter la libéralisation du commerce, améliorer la compétitivité des entreprises, augmenter l'investissement, y compris étranger, dans les secteurs productifs, etc.);

- le développement des services de soutien au commerce (accès aux crédits commerciaux, soutien aux entreprises, utilisation de technologies de l'information, conseil sur la création de nouveaux produits, contrôle de qualité, amélioration de la commercialisation et des réseaux de distribution);

- le renforcement des capacités de facilitation des échanges (modernisation des services douaniers, simplification des formalités d'exportation et d'importation);

- la formation et la mise en valeur des ressources humaines.

Les activités d'assistance technique seront précédées par un appui dans l'évaluation des besoins établis par chacun des PMA. Le cadre intégré doit également permettre aux six organisations concernées de travailler en étroite coordination afin d'éviter tous chevauchements et doubles emplois, ainsi que de programmer

14. Organisation mondiale du commerce, Comité du commerce et du développement, «Rapport de la réunion de haut niveau en faveur des PMA des 27 et 28 octobre 1997 », WT/COMTD/12, 12 novembre 1997.

15. Pour plus de détails concernant la révision du schéma de préférences douanières, se référer au chapitre «Préférences douanières», p. 284 du présent Annuaire.

16. Organisation mondiale du commerce, Réunion de haut niveau en faveur des PMA, « Présentation du cadre intégré pour l'assistance technique liée au commerce », WT/LDC/HL/1, 10 octobre 1997. 
et synchroniser convenablement l'assistance technique liée au commerce et fournie à chacun des PMA.

L'adoption à l'unanimité à l'issue de la Conférence du cadre intégré en constitue le résultat essentiel. La Suisse, qui a participé très activement à l'élaboration du document, s'est montrée très satisfaite de son adoption. Elle souligne toutefois que cette approche demeurera inopérante tant qu'elle ne sera pas adoptée par les différentes organisations internationales concernées. Dans cette optique, la Suisse s'emploiera à encourager ce processus auprès des diverses institutions concernées (OMC, PNUD, CNUCED, Banque mondiale, FMI, CCI, ONUDI). De plus la Suisse s'engage à suivre attentivement l'application du cadre intégré à ses pays de concentration.

\section{Symposium préparatoire des $O N G$}

Le manque de transparence et la non-participation des organisations non gouvernementales de développement, d'environnement, de consommateurs, des syndicats, des mouvements paysans, etc., dans le cadre des négociations de l'Uruguay Round avaient fait l'objet de nombreuses critiques. A l'occasion de la $1^{\text {re }}$ Conférence ministérielle de l'OMC à Singapour, la Communauté de travail a mené une enquête sur la représentativité de l'OMC et de ses pays membres par rapport aux Parlements nationaux et à la société civile. L'enquête a couvert 30 membres de l'OMC, dont les Etats-Unis, l'Union européenne, l'Inde, le Bangladesh, la Sierra Leone et le Nicaragua. L'article suivant en présente les conclusions et recommandations principales ${ }^{17}$. En Suisse la Communauté de travail avait coordonné un appel signé par 129 parlementaires, soit la majorité du parlement, pour demander d'améliorer la transparence de l'OMC et faciliter la participation des ONG. Dans le même but le Centre international du commerce et du développement durable (CICDD) a été créé en 1996, avec pour objectif de favoriser le dialogue entre l'OMC et la société civile et de promouvoir le développement durable dans le commerce international.

Dans le cadre de la préparation de la réunion de haut niveau pour favoriser le commerce avec les PMA, le secrétariat de l'OMC a organisé un symposium à l'intention d'une trentaine d'ONG, triées sur le volet, du Nord et du Sud. La Communauté de travail a participé au symposium en présentant une contribution sur la contribution des ONG suisses de développement au renforcement des capacités commerciales ${ }^{18}$. Ce document expose notamment l'exemple des réalisations de la Fondation Max Havelaar et présente les propositions de la Communauté de travail pour l'amélioration de l'accès aux marchés du Nord pour les exportations des PMA.

La $2^{\mathrm{e}}$ Conférence ministérielle de 1'OMC se tiendra à Genève du 18 au 20 mai 1998 , conjointement avec une réunion destinée à célébrer le $50^{\mathrm{e}}$ anniversaire du système commercial multilatéral.

17. Le texte complet a été publié dans «Accountability of the World Trade Organization", Christophe Bellmann et Richard Gerster, Journal of World Trade, décembre 1996. Une version complète est aussi disponible sur demande en français auprès de la Communauté de travail, avenue de Cour 1, 1000 Lausanne 13, tél. 021/612.00.95.

18. Communauté de travail, Contribution des ONG suisses de développement, "Renforcement des capacités commerciales des PMA», octobre 1997. Disponible sur Internet: http://www. swisscoalition. ch 


\section{SOURCES}

Organisation mondiale du commerce, Focus, Bulletin mensuel d'information de l'OMC, 1997.

Organisation mondiale du commerce, Communiqués de presse, 17 février 1997 - Press/76 «Conclusion d'un accord sur les télécommunications», 3 mars 1997 - Press/69 «Accord sur les technologies de l'information», 27 mars 1997 Press/70 «Selon R. Ruggiero, l'instauration du libre-échange pour les produits informatiques va faciliter la vie quotidienne des consommateurs et des entreprises», 27 octobre 1997 - Press/82 «A New Partnership against Marginalization».

Département fédéral de l'économie publique, Communiqué de presse, Berne, 19 novembre 1997 «Ajustement aux accords de l'OMC».

«Rapport sur la politique économique extérieure $97 / 1+2$ » du 19 janvier 1998 in Feuille Fédérale, n9, 10 mars 1998 (message 97.090).

Communauté de travail, Contribution des ONG suisses de développement, «Renforcement des capacités commerciales des PMA», octobre 1997.

Société pour le développement de l'économie suisse, Opinion, «Pour une libéralisation des télécommunications», 25 février $1997, \mathrm{n}^{\circ} 9 \mathrm{a}$.

L'Agefi, 12 mars 1997 «Le nouveau paysage économique de l'infocommunication», 27 mars 1997 «ATI, 39 pays von supprimer leurs droits de douane», 28 octobre 1997 «Les riches au chevet du Tiers Monde», 12 décembre 1997 «Jour J pour la libéralisation des services financiers» et «Les négociations de l'OMC entre les mains d'AIG», 15 décembre 1997 «L'OMC réussit la libéralisation des services financiers».

Journal de Genève, supplément spécial «Jeudi économie» du 12 décembre 1996 «Télécommunications: l’heure des choix est arrivée», 15 février 1997 «Jour J pour l'accord mondial sur la libéralisation des télécoms», 17 février 1997 «Accord de libéralisation signé à l'OMC: les géants des télécoms jubilent», 7 mars 1997 «La longue marche de la Chine vers l'OMC», 29 octobre 1997 «L'OMC veut favoriser l'intégration des pays les moins avancés».

La Liberté, 18 février 1997 «Les progrès des télécommunications facilitent la mondialisation de l'économie», 15 décembre 1997 «Un accord historique a été signé sur la libéralisation des services financiers».

Neue Zürcher Zeitung, 17. Februar 1997 «WTO-Liberalisierungspaket im Telekom-Bereich», 27. Marz 1997 «Informationstechnologie-Abkommen unter Dach», 25. September 1997 «Aufbruchstimmung in der Telekommunikation», 27. Oktober 1997 «Bündelung der Kräfte für die Dritte Welt», 15. Dezember 1997 «Erweitertes WTO-Paket für Finanzdienste».

\section{SITES INTERNET}

Organisation mondiale du commerce: http://www. wto. org/

Centre international du commerce et du développement durable: http://www. icstd. org

\section{ADRESSES UTILES}

Organisation mondiale du commerce, rue de Lausanne 154, 1211 Genève 21, tél. 022/739.51.11.

Centre international du commerce et du développement durable (International Center for Trade and Sustainable Development - ICTSD), chemin des Anémones 13, 1219 Genève, tél. 022/979.94.92, fax 022/979.90.93.

\subsection{CONFÉRENCE DES NATIONS UNIES SUR LE COMMERCE ET LE DÉVELOPPEMENT}

Lors de la 9 e Conférence sur le commerce et le développement qui s'est tenue à Midrand, en Afrique du Sud, en avril 1996, d'importantes réformes ont été décidées. La mise en æuvre de celles-ci s'est poursuivie au cours de l'année 1997. La Suisse, qui a appuyé ces réformes, travaille à leur consolidation et au renforcement de l'approche libre-échangiste pour la résolution des problèmes qui se posent dans le domaine du commerce. Elle s'engage également pour que l'organisation devienne un forum de discussion pour permettre aux Etats de débattre des difficultés que rencontrent les pays en développement dans le cadre de la globalisation.

\section{$\square$ Coopération technique}

Le Conseil du commerce et du développement a adopté, en juin 1997, une stratégie sur les activités de coopération technique qui restera en vigueur jusqu'à la tenue, en l'an 2000, de la CNUCED X. Cette stratégie a principalement pour but de renforcer les capacités humaines et institutionnelles des pays en développement et des économies en transition afin qu'ils puissent promouvoir leur propre processus de développement. 
En décembre 1996, la Suisse et la CNUCED ont signé un accord établissant un fonds d'affectation spéciale afin de financer des projets de coopération technique ayant trait au commerce. La Suisse s'est engagée pour un montant initial de 3 millions de dollars, dont un million sera consacré au fonds d'affectation spéciale pour les PMA. En 1997, la Suisse a travaillé à la mise en œuvre du fonds et à la définition de projets.

\section{$\square$ Fonds d'affectation spéciale pour les PMA}

Grâce aux contributions et aux engagements fermes des pays développés et en développement un fonds d'affectation spéciale pour les pays les moins avancés (PMA) a pu être établi en février 1997. Ce fonds, une initiative de la CNUCED, a pour but d'aider les PMA à s'intégrer dans l'économie mondiale. L'un de ses aspects novateurs est la participation d'autres financeurs que les donateurs traditionnels: les pays en développement ainsi que le secteur non gouvernemental, ONG, fondations, sociétés privées.

\section{$\square$ Investissements étrangers directs}

La CNUCED a poursuivi ses travaux d'analyses sur les investissements étrangers directs (se référer au chapitre sur lès relations financières internationales, p. 135). Dans ce but elle a organisé un séminaire pilote sur la mobilisation des investisseurs privés dans les pays en développement, qui sera suivi en novembre 1998 par un sommet qui se tiendra à Lyon sous le titre «Partenaires pour le développement». Sommet pendant lequel une large participation du secteur privé est escomptée.

A ASTM 1997, «CNUCED IX», pp. 37-43.

\section{SOURCES}

«Rapport sur la politique économique extérieure $97 / 1+2 »$ du 19 janvier 1998 in Feuille fédérale, nº, 10 mars 1998 (message 97.090).

Conférence des Nations Unies sur le commerce et le développement, Communiqués de presse, 20 décembre 1996 «La Suisse verse 3 millions de dollars à la CNUCED», 3 février 1997 «Le fonds d'affectation spéciale pour les PMA est opérationnel», 27 juin 1997 «Le conseil de la CNUCED adopte une stratégie sur la coopération technique», 25 août 1997 «L'économie mondiale tourne encore à bas régime malgré quelques éclaircies au Nord et au Sud», $1^{\text {er }}$ septembre 1997 «L'effondrement et la défaillance de l'Etat dans les PMA requièrent d'urgence l'attention internationale».

Conférence des Nations Unies sur le commerce et le développement, Rapport sur le commerce et le développement 1997, Genève, CNUCED, 1997.

Conférence des Nations Unies sur le commerce et le développement, Rapport sur les pays les moins avancés 1997, Genève, CNUCED, 1997.

L'Agefi, 16 septembre 1997 «Rapport de la CNUCED, l'écart se creuse entre les riches et les pauvres».

Neue Zürcher Zeitung, 16. September 1997 «Wachsende wirtschaftliche Disparitäten», 25. Oktober 1997 «UnctadBericht über die Ärmsten der Dritten Welt».

SITE INTERNET

CNUCED: http://www. unicc. org/unctad/fr 


\subsection{COMMERCE ÉQUITABLE}

Lancé en 1964, lors de la $1^{r e}$ Conférence de la CNUCED, l'appel "Trade, not aid» aura durablement marqué les esprits. La mobilisation de citoyens a permis l'émergence d'un important mouvement social, en Europe et en Suisse, visant à promouvoir l'idée d'un commerce plus juste. De nombreuses organisations de commerce équitable sont nées afin de combattre la précarité dans le Sud. Basées sur le concept de l'échange et non pas de l'assistance, ces associations tentent d'améliorer la situation des plus pauvres de manière durable par le biais du commerce dit équitable, du lobbying politique et de l'information aux consommateurs. Si le commerce équitable reste marginal par rapport aux flux commerciaux mondiaux, l'intérêt des consommateurs pour ce type d'échange ne cesse de grandir.

Le commerce équitable s'appuie, tant au Nord qu'au Sud, sur des mouvements organisés qui tentent d'apporter des solutions concrètes à des situations difficiles et qui contribuent à l'évolution des mentalités (sur la notion de développement, la nécessité d'une plus grande solidarité, la définition et la mise en œuvre de critères éthiques, le rôle des femmes, etc.). Le mouvement du commerce équitable cherche à établir des ponts entre les hommes, expression d'une solidarité directe entre les peuples et établissement d'un réseau original de relations transnationales. De plus, le commerce équitable offre à plus de 50'000 bénévoles en Europe le moyen de concrétiser un engagement citoyen (1500 personnes sont rémunérées dans le mouvement, soit 3\% des militants).

\section{$\square$ Les critères du commerce équitable}

Les méthodes de commercialisation équitable comprennent un certain nombre de critères éthiques:

- les produits sont achetés le plus directement possible aux producteurs dans le but de limiter le nombre d'intermédiaires;

- les producteurs sont organisés en coopérative, en association de travail communautaire ou en groupement paysan;

- les méthodes de production doivent remplir des critères écologiques;

- le prix d'achat est fixé selon le coût des matières premières et de la production, et est également fonction du temps et de l'énergie investis. Il doit permettre aux producteurs d'atteindre un niveau de vie décent. Les prix sont supérieurs au prix du marché, quel que soit le cours des matières premières par exemple; de plus un prix minimum est toujours garanti. Par ce «surprix», les producteurs sont appelés à diversifier leur production et non pas à augmenter le rendement du produit concerné;

- les producteurs ont droit à un préfinancement partiel;

- la collaboration est maintenue à long terme;

- des contacts réguliers assurent un retour d'information aux partenaires-producteurs à propos de la qualité des produits, de l'emballage, etc.;

- un monitoring (suivi et contrôle) est assuré par les associations de commerce équitable; 
- si nécessaire, des avis ou une aide sont proposés dans le domaine du développement du produit, du financement, de l'organisation, de la formation et du management. Une information sur le fonctionnement du commerce international est également dispensée aux producteurs afin de leur apporter une meilleure connaissance des marchés mondiaux des produits les concernant.

\section{$\square$ Rôle du consommateur}

Le succès du commerce équitable s'explique par la valeur ajoutée «solidarité» à laquelle une partie des consommateurs occidentaux est de plus en plus sensibilisée. L'acheteur souhaite des garanties concernant la qualité du produit, les conditions dans lesquelles il a été fabriqué ou cultivé, tant d'un point de vue social qu'écologique. Les produits, qu'ils soient alimentaires ou artisanaux, peuvent s'acheter dans des magasins spécialisés (les Magasins du Monde) ou sont certifiés par un label que le consommateur peut identifier facilement dans les grandes surfaces. D'un prix généralement plus élevé, les produits issus du commerce équitable garantissent aux clients que la plus-value sert directement à l'amélioration des conditions de vie des producteurs, par exemple en mettant celui-ci à l'abri des fluctuations des cours mondiaux du café.

Les labels du commerce équitable garantissent des critères qui peuvent légèrement varier en fonction des associations ou des produits, mais dans tous les cas ils visent:

• à garantir aux producteurs un salaire décent et un bon cadre de vie pour les travailleurs et leurs familles. Les organisations de producteurs offrent généralement un encadrement social, l'accès à un dispensaire et à une école;

• le respect de standards écologiques pour la qualité des produits. Des mesures incitatives, par un bonus financier, encouragent également les producteurs à préférer une culture biologique;

- à offrir aux consommateurs une information sur la situation dans les coopératives, les critères du commerce équitable, la vérification des critères des labels, l'évolution du commerce international, etc.

La part du commerce équitable est insignifiante comparativement à l'ensemble du commerce mondial, mais son importance grandit, ainsi que la diversité des produits répondant à ses critères. Cependant, certaines catégories de produits atteignent de 5 à $10 \%$ des parts de marché de produits concernés (voir ci-dessous le paragraphe Max Havelaar). Aujourd'hui, le commerce équitable au niveau européen atteint un chiffre d'affaires de 400 millions de francs assurant un revenu à 5 millions de personnes dans les coopératives d'Afrique, d'Amérique latine et d'Asie.

\section{$\square$ Faits marquants du commerce équitable en Suisse}

Le bilan du commerce équitable en Suisse en 1996 et 1997 est particulièrement réjouissant. Comme détaillé ci-dessous, les parts de marché du commerce équitable se sont étendues et de nouvelles initiatives sont apparues. Le chiffre d'affaires global du commerce équitable en Europe est estimé à 400 millions de francs. En 1994, la European Fair Trade Association a estimé que la Suisse arrivait en troisième position européenne avec un chiffre d'affaires de plus de 45 millions de francs suisses par an. En moyenne, chaque Suisse a acheté pour 7,50 
francs de produits «équitables» par an, ce qui le place en tête des pays européens ${ }^{19}$. L'implantation de points de vente sur tout le territoire national (magasins de commerce équitable, stands de rue tenus par des bénévoles, ventes dans les paroisses, etc.) explique cet excellent résultat. La campagne annuelle (1997) des œuvres d'entraide Pain pour le prochain et Action de Carême, sous le slogan «La bourse pour la vie», était consacrée au commerce équitable.

\section{Max Havelaar}

Dans son rapport annuel 1996, la fondation Max Havelaar dresse un bilan «très positif» de son cinquième exercice. Dans les faits, la progression du volume des ventes de produits Max Havelaar a connu une faible progression de 1\%, en dépit, il est vrai, du recul général du commerce de détail. En cinq ans Max Havelaar a lancé cinq produits sur le marché suisse. Les ventes de café labellisé, premier produit lancé en 1992, ont atteint $5 \%$ des parts de marché du produit, maintenant ainsi leur position. Le thé, quant à lui, a conquis $2 \%$ des parts de marché. En 1996, la vente de miel a enregistré un recul de 4\%, atteignant un volume de 200 tonnes. La récolte record de 1995 en Suisse a sans doute contribué à une diminution générale des importations de miel étranger. Durant la même année, le chocolat $\mathrm{a}$, en revanche, bénéficié d'une hausse des ventes correspondant à la vente de 2 millions de plaques. 1997 aura également été marquée par l'introduction des produits Max Havelaar auprès de consommateurs institutionnels; les cafétérias de certains établissements scolaires, du CERN, du CICR, de Novartis, du Palais fédéral et chez Swissair servent maintenant du café «équitable».

En mars 1997, la fondation Max Havelaar a mis sur le marché son premier produit frais: la banane. Les bananes proviennent de petits producteurs ainsi que de plantations moyennes en Equateur. Contrairement à d'autres produits Max Havelaar, dont le prix est légèrement plus élevé pour le consommateur, celui de la banane correspond aux prix des fruits provenant des entreprises multinationales. En une année la banane aura conquis $13 \%$ des parts de marché. Deux nouveaux produits, le jus d'orange et les fleurs coupées, sont à l'étude pour être mis en vente en automne 1998 et 1999.

Lancée en 1992, la fondation se finance principalement par les recettes liées à l'octroi de la licence Max Havelaar, la contribution des œuvres d'entraide fondatrices et l'Office fédéral des affaires économiques extérieures (OFAEE). Cependant les membres fondateurs escomptaient que la fondation puisse atteindre son autonomie financière en cinq ans. Ce n'est, pour l'instant, pas le cas, l'autofinancement ayant pourtant évolué positivement en 1996, passant de $53 \%$ à $66 \%$. La fondation prévoit un autofinancement à $75 \%$ pour fin 1997 , grâce au succès de la banane. Afin d'étudier l'opportunité de reconduire son soutien, l'OFAEE a mandaté l'Institut universitaire d'études du développement pour évaluer les conséquences d'une diminution, voire d'une suppression, des subventions. Le rapport ${ }^{20}$ fournit une étude d'impact du label en Suisse et dans les pays producteurs et détaille la filière de commercialisation du café. Dans leurs conclusions, les auteurs reconnaissent que la mise en place d'un label coûte cher et encouragent les organisations fondatrices et la Confédération au maintien de

19. European Fair Trade Association, Commerce équitable - Mémento 1995, EFTA, octobre 1995, p. 35.

20. Institut universitaire d'études du développement, Evalutation du soutien de l'OFAEE à la fondation Max Havelaar dans le cadre de l'aide au développement, rapport rédigé sous la direction de Claude Auroi à la demande de l'OFAEE, Genève, IUED, novembre 1996. 
leur engagement financier selon un plan dégressif. Les recommandations à l'égard de la fondation portent sur trois points essentiels: la nécessité de consolider les parts de marché des produits déjà lancés, le besoin d'une évolution vers l'autonomie financière et enfin une plus grande prudence dans le lancement de nouveaux produits, chacun d'entre eux demandant de gros efforts de gestion.

\section{OS3 - Claro}

1997 aura marqué un tournant important dans la distribution du commerce équitable en Suisse. En octobre 1997, une société anonyme, Claro SA, a remplacé la coopérative d'importation OS3, fondée en 1977 par les œuvres d'entraide. La coopérative commercialisait plus de 700 articles, matières premières et produits finis ou semi-finis, dans les domaines de l'alimentation et de l'artisanat, marchandises fournies par 110 producteurs en provenance de 37 pays, selon des critères sociaux et écologiques. Les principaux clients d'OS3 étaient les Magasins du Monde, des commerces biologiques et alternatifs, ainsi que des petits détaillants de quartier répartis dans toute la Suisse. Pour son dernier exercice juin 1996-1997, la coopérative OS3 affichait un chiffre d'affaires d'un peu plus de 11 millions de francs.

Après l'introduction de produits Max Havelaar dans l'assortiment de grands distributeurs telles la Migros et la Coop, la nécessité d'une restructuration s'est faite de plus en plus forte au sein des organisations du commerce équitable. La volonté initiale était de créer une organisation nationale, afin de renforcer l'identité commune du mouvement, de rationaliser la distribution (jusqu'alors divisée entre une centrale d'importation et sept grossistes) et de conquérir de nouvelles parts de marché.

Lors d'une assemblée extraordinaire de la coopérative en février 1997, la transformation d'OS3 en Claro SA a été approuvée à la quasi-unanimité, l'Association romande des Magasins du Monde s'étant abstenue. Cette abstention marque une divergence idéologique importante entre la Suisse romande, qui souhaite maintenir une dimension associative et politique au mouvement, et la Suisse alémanique, qui souhaite doter le commerce équitable de moyens de gestion plus conventionnels: renforcement du marketing, image de marque commune et professionnalisation des ventes. Dans les faits 130 magasins à l'enseigne "Claro" ont été rebaptisés du côté alémanique; du côté romand les Magasins du Monde conservent leur logo, mais iront s'approvisionner auprès de la centrale de distribution Claro.

\section{STEP}

Après deux ans d'activité, la fondation STEP dresse un bilan intermédiaire globalement positif. Fondée en 1995 par des œuvres d'entraide ${ }^{21}$ et IGOT, l'association suisse pour un commerce loyal de tapis d'Orient, la fondation s'engage à améliorer les conditions de travail et le respect de l'environnement dans la production de tapis, avec une attention particulière contre le travail abusif des enfants. Elle surveille le respect du code de conduite en Suisse et dans les pays producteurs, grâce à l'appui d'experts indépendants. De plus STEP soutient sept projets, en Inde et au Népal, visant à améliorer l'autonomie et la situation sociale des personnes défavorisées. 
INITIATIVES EN SUISSE POUR UN COMMERCE ÉQUITABLE

\begin{tabular}{ll}
1964 & $\begin{array}{l}\text { Création de la CNUCED à l'initiative des pays en développement. } \\
\text { Son but: des échanges plus justes. Son slogan: Trade, not aid. }\end{array}$ \\
\hline 1973 & Caritas ouvre les Fairness Shops et Gebana importe des bananes d'Amérique centrale. \\
\hline 1974 & $\begin{array}{l}\text { Ouverture du premier Magasin du Monde en Suisse romande; aujourd'hui il y a plus de } \\
1000 \text { points de vente dans le pays. }\end{array}$ \\
\hline 1977 & $\begin{array}{l}\text { OS3, coopérative d'importation des produits équitables, est fondée par } 18 \text { œuvres d'en- } \\
\text { traide. }\end{array}$ \\
\hline 1983 & $\begin{array}{l}\text { Début du processus de négociation entre la Migros, Del Monte et des œuvres d'en- } \\
\text { traide suisses pour établir une clause sociale garantissant des conditions de travail et } \\
\text { des salaires supérieurs dans les plantations d'ananas aux Philippines. }\end{array}$ \\
\hline 1986 & $\begin{array}{l}\text { Lancement d'une pétition «Pour un commerce équitable avec le Tiers Monde» par la } \\
\text { Communauté de travail des œuvres d'entraide, pétition qui a récolté plus de 110'000 } \\
\text { signatures. Le succès de cette pétition a été un des éléments de la mise en place du } \\
\text { label Max Havelaar. }\end{array}$
\end{tabular}

\begin{tabular}{ll}
\hline 1992 & $\begin{array}{l}\text { La fondation Max Havelaar est créée en Suisse. La même année, lancement du café } \\
\text { Max Havelaar. Pour la première fois des "produits certifiés équitables" grâce au label } \\
\text { font leur entrée dans les grandes surfaces. La vente de café Max Havelaar atteint 5\% } \\
\text { des ventes de café. } \\
\text { Nouveaux produits: } 1993 \text { - miel, 1994 - chocolat, 1995 - thé, 1997 - bananes. }\end{array}$ \\
\hline 1995 & Création des labels STEP (tapis) et DIP (textile). \\
\hline 1996 & TerrEspoir (importation directe de produits frais d'Afrique). \\
\hline Hiver 1996-97 & $\begin{array}{l}\text { Le commerce équitable se soucie de plus en plus des conditions de travail dans l'in- } \\
\text { dustrie. Lancement par la Déclaration de Berne et Terre des hommes-Suisse de la cam- } \\
\text { pagne Let's go fair, demandant aux entreprises d'Asie du Sud-Est, ainsi qu'à leurs } \\
\text { sous-traitants, le respect de codes de conduite dans les usines de production de chaus- } \\
\text { sures de sport. } \\
\text { Campagne annuelle de Pain pour le prochain et Action de Carême consacrée au com- } \\
\text { merce équitable. }\end{array}$ \\
\hline Octobre 1997 & $\begin{array}{l}\text { Remplacement de la coopérative d'importation de produits du Sud OS3 par une société } \\
\text { anonyme, Claro. }\end{array}$ \\
\hline
\end{tabular}

Source: Nouveau Quotidien, supplément 13 février 1997 "Le commerce change le monde".

Les importateurs ou les commerçants de tapis signent un contrat de licence avec STEP, ce qui leur permet d'afficher le label de la fondation. En contrepartie ils s'engagent à respecter un code de conduite définissant des conditions de production socialement équitables et respectueuses de l'environnement. Les preneurs de licence versent une contribution de 4 francs par mètre carré de tapis vendu à STEP.

En deux ans STEP a signé des contrats avec 12 preneurs de licence, qui représentent 12 points de vente et $23 \%$ du marché suisse. En septembre 1997, une première filiale du groupe Migros, Migros Bâle, a signé un contrat. De plus, depuis avril 1997, l'Office fédéral des affaires économiques extérieures soutient financièrement la fondation. 


\section{TerrEspoir}

Depuis 1992, TerrEspoir importe des produits agricoles d'Afrique. De moins de 100 kilos par semaine à ses débuts, l'organisation a importé environ 2,5 tonnes par semaine d'une trentaine de fruits et légumes tropicaux frais ou transformés en 1997. Etablie en tant que fondation en 1996 par le Département missionnaire et Pain pour le prochain, TerrEspoir est au service du commerce équitable entre la Suisse et l'Afrique. Elle soutient des groupements de petits cultivateurs africains à travers la commercialisation de leurs produits.

CD ASTM 1996, «Expériences de Max Havelaar...», R. Buser et M. Schmutz Cattaneo, pp. 257-264.

CD ASTM 1997, «Production et commerce international...», D. Rüesch, pp. 229-246.

\section{SOURCES}

European Fair Trade Association, Commerce équitable-Mémento 1995, EFTA, octobre 1995.

Pain pour le prochain, Repères G 2/98, «Mondialisation, inégalités et commerce équitable», printemps 1998.

Communauté de travail, Contribution des ONG suisses de développement, «Renforcement des capacités commerciales des PMA», octobre 1997.

Office fédéral des affaires économiques extérieures, document de travail «Politique de l'OFAEE en matière de déclarations de labels", mars 1997.

Fondation Max Havelaar, Dossier de presse, 18 mars 1997 «La banane au label Max Havelaar», et Rapport annuel 1996, mars 1997.

Association romande des Magasins du Monde, Forum MdM, paraît quatre fois par an.

OS3, Rapports d'activité, 1995-96 et 1996-97, parution en juin.

Claro AG, Unterlagen für die Medien, 17. Oktober 1997.

STEP, Communiqué de presse, 28 octobre 1997.

Andràs November, in Choisir, février 1997, «Le commerce équitable ou l'éthique de la consommation».

La Liberté, 4 novembre 1996 «L'art de vendre le parfum de l'Afrique», 27 janvier 1997 «L'éthique des étiquettes progresse».

Le Courrier, 8 février 1997 «Reportage et études autour du symbole reconnu du commerce équitable».

Mosquito, Nr. 8 Dezember 1996 «Fairer Handel zwischen Gerechtigkeit und Markt».

Nouveau Quotidien, supplément du 13 février 1997 «Le commerce change le monde».

Neue Zürcher Zeitung, 13. Februar 1997 «Der faire Handel mit Dritteweltprodukten im Umbruch».

Sonntagszeitung, Februar 1997 «Fair Handelszeitung», 16 pages.

\section{SITE INTERNET}

TerrEspoir: http://www. megaphone. ch/terrespoir/

\section{ADRESSES UTILES}

Association romande des Magasins du Monde, avenue de Genève 52, 1004 Lausanne, tél. 021/661.27.00, fax $021 / 661.22 .20$

Claro SA, Byfangstrasse 19, 252 Orpund, tél. 032/356.07.00, fax 032/356.07.01

Fondation Max Havelaar, 1309 Cuarnens, tél. et fax 021/864.41.46.

STEP, Monbijoustrasse 31, case postale 8348, 3001 Berne, tél. 031/382.77.88, fax 031/382.75.85.

TerrEspoir, case postale 67, 1373 Chavornay, tél. 024/441.73.42, fax 024/441.79.76. 